\title{
Morphogenetic processes in animals and plants
}

\author{
Published online: 19 November 2010 - C EDP Sciences / Società Italiana di Fisica / Springer-Verlag 2010
}

Living tissues and organisms are made of large numbers of cells which are closely packed together to form complex morphologies. Tissue structures in animals are formed by developmental processes when a fertilized egg divides and subsequent divisions create large numbers of cells that organize in space and time. Supracellular structures can also develop later in the life of organisms, for example, during the growth of tumours or, as is the case for plants, depending on seasonal cues. The mechanisms underlying such pattern formation have long been studied in developmental biology. The revolution of molecular biology and cell biology has led to a number of ground-breaking insights and produced a wealth of new data. It is becoming clear that in order to integrate the many molecular players and to extract key mechanisms that underlie structure formation on large scales requires an interdisciplinary approach. Physical concepts will likely play a major role in this context.

From a physicist's point of view, developing tissues represent an extraordinarily complex form of soft matter. Their inherent activity and intercellular communication pose enormous challenges to describe the mechanical properties of these systems. For example, cell division and growth are important active processes that lead to the generation of stresses in a cell ensemble: growing cells need space and push their neighbours aside, dividing cells often change their morphology, which also influences adjacent cells as their are connected. In the same vein, programmed cell death (apoptosis) is an active process changing the mechanical conditions in a developing tissue. The ensuing stresses as well as cell crawling lead to a dynamic reorganization of tissues. On top of these purely mechanical aspects, cells communicate with each other through molecular signalling systems. Descriptions of active mechanical properties together with the chemical signals present during the formation of spatial morphologies require new concepts in order to account comprehensively for the numerous processes present on largely different length and time scales.

This special issue highlights current approaches to study dynamic tissues as physical systems and as soft materials. General physical properties are studied in the work by Klopper et al. on the sorting of cell aggregates and by Kaschube et al. on contractile cytoskeletal meshworks. Processes in the fruit fly, one of the key model organisms studied in developmental biology, are the subject of the contributions of Aegerter et al. on photo-elastic properties of the fly's wing precursor, of Staple et al. on a vertex model for studying physical properties of epithelial cell packing and by Lubensky et al. on the development of the fly's intricate eye structure. Rieger et al. address the physical principles underlying the remodeling of the vascular network during tumour growth, while Alim et al. consider the formation of veins in plant leaves. Together, these works provide a taste of the rich dynamic behaviour and the unconventional mechanical properties of living multicellular systems and illustrate how physical approaches can significantly contribute to a deeper understanding of these fascinating processes. We expect that new physical mechanisms of patterning will be discovered by the study of developmental processes as physical systems. 